CIC. Cuadernos de Información y Comunicación

ISSN: 1135-7991

https://dx.doi.org/10.5209/ciyc.75818

\title{
El audio efímero como comunicación digital innovadora. El caso Clubhouse $^{1}$
}

\author{
Eglée Ortega Fernández², Graciela Padilla Castillo ${ }^{3}$, Jonattan Rodríguez $^{4}$
}

Enviado: 01/05/2021 / Evaluado: 11/05/2021 / Aceptado: 12 / 05/2021

Resumen. La red social Clubhouse es un fenómeno sin precedentes, por su rápido crecimiento en un único año de vida y por sus originales características. Hasta mayo de 2021, sólo podía ser descargada en un dispositivo con sistema iOS y previa invitación privada de un usuario registrado. Esta investigación ahonda en sus claves, usos y posibilidades a través de una metodología correlativa: 1) Investigación bibliográfica longitudinal sobre los conceptos de conectividad, fenómeno Clubhouse, y sus antecedentes, problemas o dilemas, tendencias y posibles cambios; 2) Análisis cuantitativo de los 50 primeros influencers de Clubhouse a partir de las categorías: Número de seguidores, Cargo/sector profesional, Nacionalidad, Generación, Sexo. Se concluye que Clubhouse tendrá que validar su espacio propio, más allá de la COVID-19 y sus limitaciones técnicas, remarcando sus fortalezas: no importan la imagen ni los likes, ofrece salas de debate íntimas, promueve discusiones no mediadas sobre temas de actualidad con líderes de opinión e influencers, y reivindica la voz como herramienta básica, accesible y universal contra la brecha digital.

Palabras clave: Redes sociales; Clubhouse; voz; audio; píldoras sonoras; innovación digital; viralidad; influencers.

\section{[en] Ephemeral audio as innovative digital communication. Clubhouse case}

\begin{abstract}
The Clubhouse social network is an unprecedented phenomenon, both for its rapid growth in just one year and for its original features. Until May 2021, it could only be downloaded on an iOS device and with a private invitation from a registered user. This research delves into its keys, uses and possibilities through a correlative methodology: 1) Longitudinal bibliographic research on the concepts of connectivity, the Clubhouse phenomenon, and its background, problems or dilemmas, trends and possible changes; 2) Quantitative analysis of the top 50 Clubhouse influencers based on the following categories: Number of followers, Position/professional sector, Nationality, Generation, Gender. It is concluded that Clubhouse will have to validate its own space, beyond COVID-19 and its technical limitations, highlighting its strengths: it is not about images or likes, it offers intimate discussion rooms, it promotes unmediated discussions on current issues with opinion leaders and influencers, and it claims the voice as a basic, accessible and universal tool against the digital divide.

Keywords: Social networks; Clubhouse; voice; audio; audio pills; digital innovation; virality; influencers.

1 Esta investigación es parte del Proyecto Innova-Docencia UCM, número 406. Título: Minivídeos en redes sociales como herramientas didácticas creativas para la igualdad. Duración: 01/10/2020-30/06/2021. Plan Estratégico del Vicerrectorado de Calidad de la UCM 2015-2019.

21egleort@ucm.es

3racielp@ucm.es

4_jonrodri@ucm.es
\end{abstract}




\section{[pt] O áudio efémero como comunicação digital inovadora. Caso Clubhouse}

Resumo. A rede social Clubhouse é um fenómeno sem precedentes, devido ao seu rápido crescimento em apenas um ano de vida e às suas características originais. Até Maio de 2021, só podia ser descarregado num dispositivo iOS e com um convite privado de um utilizador registado. Esta investigação aprofunda as suas chaves, usos e possibilidades através de uma metodologia correlativa: 1) Pesquisa bibliográfica longitudinal sobre os conceitos de conectividade, fenómeno Clubhouse, e o seu contexto, problemas ou dilemas, tendências e possíveis mudanças; 2) Análise quantitativa dos 50 principais influenciadores Clubhouse com base nas categorias: Número de seguidores, Posição/sector profissional, Nacionalidade, Geração, Género. Conclui-se que o Clubhouse terá de validar o seu próprio espaço, para além da COVID-19 e das suas limitações técnicas, destacando os seus pontos fortes: independentemente da imagem ou do gosto, oferece salas de discussão íntima, promove discussões não mediadas sobre questões actuais com líderes de opinião e influenciadores, e reivindica a voz como uma ferramenta básica, acessível e universal contra a fractura digital.

Palavras-chave: Redes sociais; Clubhouse; voz; áudio; comprimidos de som; inovação digital; viralidade; influenciadores.

Sumario. Introducción: ¿por qué estudiar el audio tras la COVID-19?. Antecedentes de las redes sociales y germen de Clubhouse. Clubhouse. Una red social para la voz. Metodología de la investigación. Resultados. 1. Conclusiones, discusión y prospectivas. Referencias Bibliográficas

Cómo citar: Ortega Fernández, E.; Padilla Castillo, G.; Rodríguez, J.(2021), El audio efímero como comunicación digital innovadora. El caso Clubhouse, en CIC. Cuadernos de Información y Comunicación 26, 107-121.

\section{Introducción: ¿por qué estudiar el audio tras la COVID-19?}

El $23^{\circ}$ Informe Navegantes en la Red, realizado por la Asociación para la Investigación de Medios de Comunicación (AIMC, 2021), detalla el crecimiento de la conectividad a Internet con una frecuencia constante, según el 47,48\% de los encuestados, y un $92,30 \%$ que se conecta varias veces al día. El smartphone es el dispositivo de acceso más elegido, según el 93,1\% de los encuestados, y el ordenador portátil (71,4\%) o sobremesa $(50,75 \%)$ ocupan respectivamente, el segundo y el tercer puesto.

De acuerdo al mismo informe, los españoles pasábamos 3 horas y 55 minutos diarios conectados a Internet. Según el $23^{\circ}$ Informe Navegantes en la Red, en 2021, este tiempo ascendió a 4 horas y 12 minutos diarios (AIMC, 2021). La actividad de los encuestados, en los últimos 30 días, refería que subieron las videollamadas $(44,7 \%)$, el uso de plataformas para el visionado de series y películas $(61,2 \%)$, la búsqueda de información sobre temas de salud (44,1\%), gestiones con la Administración (49,0\%) y escucha de música online $(67,1 \%)$. Sin duda, son acciones que nos recuerdan a la crisis socio-sanitaria de la COVID-19. Sin embargo, más allá del cambio que ha supuesto el virus en nuestras vidas, esta encuesta se realizó después del confinamiento y segunda ola de infecciones en España, y se refiere a nuevos hábitos y usos que parecen no responder solamente a esa crisis temporal.

Por ejemplo, podemos centrarnos en la subida exponencial de las videollamadas. Ofrecieron una solución laboral, educativa, familiar y social durante el confinamiento, donde no existía ninguna posibilidad de desplazamiento. Cuando las medidas de distanciamiento se redujeron, las videollamadas siguen estando presentes por las posibilidades que ofrecen. AIMC (2021) ordena las más elegidas, en orden decreciente: 
WhatsApp (67,6\%), Zoom (42,5\%), Microsoft Teams (30,7\%) y Skype (29,7\%). Del mismo modo, proliferan los estudios sobre la importancia terapéutica de esas llamadas en concreto y de la voz humana en general (Mills, Stoneham \& Georgiadou, 2017; Grier, 2019; Quinn, McGilloway \& Burke, 2021; Stevenson, 2021).

Teniendo en cuenta también que un $87 \%$ de los internautas de entre 16 y 65 años utiliza redes sociales (IAB Spain, 2020a), podemos deducir que gran parte de ese tiempo de conexión se dirige a estas plataformas nacidas para socializar, pero desarrolladas y evolucionadas hacia el marketing y la interactividad en todo momento. En este Estudio de Redes Sociales 2020 (IAB Spain, 2020a) existe una categorización que indica las actividades más realizadas por los usuarios. Un $81 \%$ busca entretenerse, un $77 \%$ pretende interactuar y un $66 \%$ procura informarse. En consecuencia, las redes sociales se habrían adaptado y evolucionado para atender positivamente todas estas necesidades y queda demostrado por el crecimiento de usuarios y por el aumento de tiempo de conexión que hemos enunciado en las primeras líneas.

Aprovechando las tecnologías y novedosas herramientas que aportan las redes sociales, los consumidores han pasado a convertirse en protagonistas y en generadores de contenidos. En 1979, Alvin Toffler, en su libro La tercera ola, planteaba que los consumidores son un fenómeno de la era industrial. El experto sostenía que en la era postindustrial, el comportamiento del consumidor avanzaba hacia ser prosumidor, aquel que además de consumir, producía por sí mismo. Más de cuarenta años después, este concepto sigue vigente y más plausible que nunca.

Verwey (2015) destaca la naturaleza colaborativa y expresiva de los medios y la tecnología interactivos, y la capacidad que ofrecen a los usuarios para participar en la producción y publicación de contenido de marca dentro de las comunidades digitales de afinidad. Añade que este panorama presenta mayores posibilidades de autoexpresión, así como oportunidades ilimitadas de participación para determinar e influir en las narrativas que se estén desarrollando a cada momento.

Marinas (2019) apunta que con las redes sociales han surgido nuevas formas de comunicación y aprendizaje hasta ahora desconocidas: "Desde expresar emociones con emoticones o emojis a recurrir a cuentas de fotos biográficas para conocer qué estaba sucediendo en un momento concreto de la historia" (Marinas, 2019, p. 187).

Al repasar la evolución de las redes sociales, podemos afirmar que éstas han pasado de la simple conexión a la promoción de auto-creación de textos, imágenes, vídeos y minivídeos. Tras la crisis de la COVID-19, el cambio de hábitos humanos en la comunicación en todos los niveles y esa recuperación terapéutica y acompañadora de la voz, como herramienta humana básica y gratuita, erigen la palabra sonora como el nuevo lenguaje digital para las redes sociales, los medios sociales, los expertos y divulgadores y por supuesto, la audiencia.

La voz es un nuevo canal conector social, internacional, abierto y accesible. La red social Clubhouse lo ha entendido y potenciado, facilitando la comunicación efímera a través de la voz, con participaciones donde la imagen y los likes quedan de lado. Esta investigación original e inédita ahonda en sus claves, usos y posibilidades.

\section{Antecedentes de las redes sociales y germen de Clubhouse}

La comunidad científica suele convenir que la primera red social del mundo fue SixDegrees, nacida en 1997 y pensada para crear listas de amigos. Se basaba en la Teo- 
ría de los seis grados de separación, que afirma que es posible conectar con cualquier persona del mundo en tan sólo 6 conexiones humanas (Bai et al., 2020).

Al repasar estudios científicos sobre los orígenes y aplicaciones de las redes sociales, destacamos a Matassi y Boczkowski (2020) y García-Jiménez et al. (2020) entre los más recientes. Mason y Narcum (2021) también han estudiado la importancia que ganaron las redes sociales dentro del marketing digital en tiempos de la COVID-19. Y dentro de este mismo marco, Al-Regaiey et al. (2021) han investigado la influencia de las redes sociales en la actitud de los padres y madres ante la administración de las vacunas.

Sobre las redes sociales y su uso por parte de estudiantes podemos mencionar a Aladro, Jivkova y Bailey (2018), López Fuentes (2020), Abelairas-Etxebarria y Mentxaka (2020), El-Khoury et al. (2020) y Chiza-Lozano et al. (2021). El-Khoury et al. (2020), concretamente, plantearon un estudio para la 'desintoxicación' por la frecuencia de uso de las redes sociales, por parte de jóvenes universitarios.

Bentley et al. (2021) desarrollaron su investigación sobre el engagement de las redes sociales como plataforma para influenciadores globales, otro punto muy interesante para analizar Clubhouse. Y Ponce (2012) menciona cronológicamente el surgimiento de las redes sociales para entender su evolución: AOL Instant Messenger (1997), Friendster (2002), My Space y LinkedIn (2003), Facebook (2004), YouTube (2005), Twitter y Tuenti (2006), WhatsApp (2009), Instagram y Pinterest (2010). Añadimos, tras éstas, TikTok (2016) y Clubhouse (2020). Aunque se podrían listar otras, nombramos las más destacadas y que han tenido mayor crecimiento desde sus inicios o sobre todo, con motivo de la crisis socio-sanitaria mundial por el coronavirus.

Sobre las más recientes, TikTok y Clubhouse, aun cuando hay pocas investigaciones académicas que las abordan como objeto de estudio, Ortega y Rodríguez (2021) y Padilla (2021) detallan que en la primera, la característica principal es el humor y la sencillez de los contenidos que atraen a los más jóvenes. Pedrero (2021) afirma que Clubhouse se centra en un público más adulto, que da más importancia a la voz que a la imagen o a los likes. Estas tendencias muestran el camino tan extenso que se ha abierto desde esa primera red social de 1997, SixDegrees, pensada para crear listas de amigos, hasta una red que nos permite conectarnos en tiempo real para hablar o escuchar sobre temas tan diversos como el marketing, el fútbol o la gastronomía, como ocurre en Clubhouse (2020).

El estudio Top Tendencias Digitales 2020 (IAB Spain, 2020b) ya detallaba varios apartados relacionados con el desarrollo y el rápido impacto de esta nueva red social. Sobre los hitos de innovación tecnológica, apuntaba que la voz se estaba integrando más en nuestras vidas, en estos momentos de híper-personalización con respecto a lo digital, gracias al uso constante del Smartphone. En estas circunstancias, las redes se decantan por contenidos más sociales, locales y personalizados.

Clubhouse tiene similitudes con la radio, como medio tradicional, y con los podcasts, como productos digitales que utilizan la voz. Sin embargo, confía en una nueva particularidad: lo efímero, ya que las conversaciones que ocurren en Clubhouse no quedan grabadas. La red social arriesga y propone un cambio de contexto y uso sin antecedentes. La nueva dinámica se centra en la comunicación que se vive en primera persona y que se establece en el aquí y en ahora.

El proceso de Clubhouse recuerda a la radio como medio tradicional, pero sin las barreras de entrada. No se necesita preparación profesional previa; sólo hay que 
estar en la sala como oyente y levantar la mano para participar directamente, cuando así lo desee el usuario. La radio en internet, como otra antecesora de esta aplicación, ha sido estudiada últimamente por Calvo y Padilla (2011), Videla y Piñeiro (2017), Pedrero y Pérez (2017), Pedrero y García (2019), Rimoldi (2020), Barbeito y Perona (2020) y Piñeiro (2021a y 2021b).

Videla y Piñeiro (2017) apuntaban, de forma providencial, que la radio online había creado un contexto de consumo híbrido, donde se unían la emisión tradicional con las nuevas formas de distribución de contenidos. En este contexto los oyentesusuarios diseñaban estrategias propias para el consumo radiofónico, seleccionando y combinando contenidos, plataformas y dispositivos de recepción. Después, el podcast se convirtió en el formato por excelencia para quienes buscan establecer dónde, cuándo y cómo consumir contenidos de audio. Están muy bien delimitados en duración y temáticas, lo que permite al usuario concretar su consumo.

Un podcast puede ser definido como un blog de audio, una radio a la carta a la que uno se suscribe mediante un sencillo sistema de datos de redifusión (en inglés, Really Simple Syndication o RSS). El término podcast surgió como contracción, llamada portmanteau en algunos ámbitos, de las palabras PoD (Personal on Demand) y broadcast (transmisión). El origen del podcasting se sitúa alrededor del 13 de agosto de 2004, cuando Adam Curry usó una especificación del formato RSS de Dave Winer, para incluir archivos adjuntos de audio (Parra, 2011).

Sobre el podcast, el estudio sobre el Top Tendencias Digitales 2020 (IAB Spain, 2020 b) apunta que es uno de los formatos que más creció en 2020, gracias a su capacidad de llegar a audiencias altamente cualificadas e interesadas en temáticas concretas. Para las marcas es un vehículo muy valioso para generar autoridad y credibilidad (Padilla, 2016; Bernárdez, Padilla y Sosa, 2019; Caldevilla, Barrientos y Padilla, 2021; Ortega y Rodríguez, 2021).

\section{Clubhouse. Una red social para la voz}

Clubhouse ya es calificado como fenómeno debido al rápido crecimiento que ha experimentado desde su lanzamiento, en abril de 2020. Un mes después de su inauguración, en mayo de 2020, recaudó 100 millones de dólares de fondos de capital riesgo y pasó de los 600.000 a los 10 millones de usuarios, entre diciembre de 2020 y marzo de 2021 (Hogan, 2021).

La red social nació de las mentes de Paul Davison y Rohan Seth, dos emprendedores con un gusto en común: los productos sociales. Se conocieron en 2011, pero fue en 2019 cuando se reencontraron y comenzaron a materializar su proyecto. Lanzaron Clubhouse en marzo de 2020, casi al mismo tiempo que la Organización Mundial de la Salud (OMS) declaraba la pandemia de la COVID-19. "Nuestro objetivo era crear una experiencia social que se sintiera más humana, una en la que, en lugar de publicar, pudieras reunirte con otras personas y hablar", señalan Davison y Seth (Paredes, 2021).

Aun cuando las investigaciones académicas sobre la red social son todavía incipientes, los medios de comunicación y especialistas en temas digitales han buscado la forma de explicar el éxito de esta red. Coinciden en que sus claves son: la expectativa por una invitación privada (no puedes crear una nueva cuenta sin invitación de otro usuario), lo variado de los contenidos (en decenas y cientos de salas, agrupadas 
por temas) y la accesibilidad que ofrece para escuchar en una misma sala, en directo y sin intermediarios, a Elon Musk, creador y director general de Tesla, Mark Zuckerberg, cofundador y director ejecutivo de Facebook, los raperos Drake y Kanye West, o la estrella mediática y empresaria, Oprah Winfrey (Lorenzo, 2021).

Clubhouse lo consigue confiando su éxito al audio. Su funcionamiento no dista mucho de cualquier otra red social, por ser gratuita, internacional, pensada sobre todo para smartphones y por requerir ciertos datos personales de sus usuarios. Hasta mayo de 2021, sí se diferenció de las demás redes porque durante más de un año, estuvo solamente disponible para el sistema operativo iOS (el que utiliza el smartphone iPhone de Apple). A partir de inicios de mayo, abrieron las descargas desde Android (sistema de código abierto presente casi todos los teléfonos que no son iPhone) en Estados Unidos y desde el resto de los países se puede apuntar a un registro previo, que avisará al usuario cuando esté disponible para descargar.

Una vez invitados, los nuevos usuarios seleccionan un identificador y configuran un perfil, de manera muy similar a Twitter. El siguiente paso es buscar y seguir amigos u otras personas de gustos similares. Una vez que se sigue a otros usuarios en Clubhouse, comienzan a aparecer los eventos que están sucediendo en la plataforma y que pueden ser de interés, de acuerdo a los gustos previamente marcados. Así, puede unirse a cualquier evento público como oyente en los llamados rooms o salas.

Las conversaciones en cada sala son gestionadas por los moderadores, que controlan a las personas que pidan la palabra, para participar en la conversación. La mayoría de los participantes dejan sus micrófonos en silencio hasta que los llama el moderador; para convertirse en participantes, los oyentes sólo tienen que levantar la mano.

La plataforma también incluye un calendario de próximos eventos, aunque cada usuario puede buscar nuevas salas y eventos por temas. Las categorías de temas populares incluyen deportes, entretenimiento, lenguaje, tecnología, bienestar y artes. Sobre el funcionamiento de la plataforma hay quienes objetan algunos detalles como que estas salas virtuales no admiten más de 5.000 participantes por ahora. Del mismo modo, Clubhouse tiene la potestad de decidir si un audio se mantiene a largo plazo y en sus condiciones y términos, impide expresamente a los usuarios grabar las conversaciones (BBC News Mundo, 2021).

Al ser una red social tan reciente e insólita, ha generado nuevas dudas sobre los niveles de privacidad. Teniendo en cuenta lo efímero del contenido basado únicamente en el audio, se podría exponer a restricciones en países como China, donde ya varias redes sociales como Twitter y Facebook están prohibidas por la imposibilidad de medir y controlar las opiniones que los usuarios comparten en ellas.

\section{Metodología de la investigación}

Tras la introducción, revisión de antecedentes de la plataforma Clubhouse y estudio de la plataforma, se han propuesto los siguientes objetivos principales para la investigación:

- Comprender los primeros pasos de Clubhouse, su nacimiento, evolución y auge hasta el momento actual.

- Conocer el contexto global y entender el fenómeno de esta red social a nivel internacional. 
- Analizar los principales influencers de la red social, examinar el volumen de followers en sus perfiles, así como la nacionalidad o sector profesional de estos creadores de contenido. Es necesario para entender qué busca la gran mayoría de los usuarios cuando acceden a esta red social en busca de contenido.

Para cumplir estos objetivos, la metodología de la investigación se basa en dos pilares correlativos, que tratan de obtener resultados inéditos y aplicables a otros trabajos académicos:

- Investigación bibliográfica longitudinal, donde se han repasado los conceptos relacionados con el estudio: crecimiento de la conectividad a Internet, fenómeno Clubhouse desde su aparición, antecedentes de la red social, problemas o dilemas éticos, tendencias y posibles cambios en la aplicación. Se ha realizado a través de fuentes bibliográficas de primer nivel: Journal Citation Reports (Social Sciences), Scimago Journal Country Rank, Dialnet, Elsevier online, SAGE Journals, Taylor and Francis Online, Dialnet y Academia.edu.

- Análisis cuantitativo de los 50 primeros influencers de Clubhouse, de acuerdo a la base de datos https://clubhouseranking.net/ y con fecha de vaciado de datos de 31 de marzo de 2021. El estudio de este medio centenar de creadores de contenido permite obtener una imagen fidedigna del contenido que se realiza en la plataforma, así como sus temáticas y tendencias. Al ser una aplicación tan reciente y de contenido tan efímero, no existe ninguna API (Application Programming Interface) que permita medir los parámetros que se necesitan para el análisis más profundo de las salas y los usuarios en las mismas.

Las categorías elegidas y estudiadas en el análisis cuantitativo de los 50 principales influencers han sido las siguientes: 1. Número de seguidores. 2. Cargo/sector profesional. 3. Nacionalidad. 4. Generación. 5. Sexo.

\section{Resultados}

Una vez establecidas las categorías, se observan los siguientes resultados en el número de seguidores:

\begin{tabular}{|c|c|c|c|c|}
\hline $\begin{array}{c}\text { Total de } \\
\text { seguidores }\end{array}$ & $\begin{array}{c}\text { Seguidores } \\
\mathbf{N}^{\mathbf{}} \mathbf{1}\end{array}$ & $\begin{array}{c}\text { Seguidores } \\
\mathbf{N}^{\mathbf{0}} \mathbf{5 0}\end{array}$ & $\begin{array}{c}\text { Diferencia entre } \\
\mathbf{e l ~}^{\mathbf{0}} \mathbf{\mathbf { y }} \mathbf{5 0}^{\mathbf{0}}\end{array}$ & $\begin{array}{c}\text { Media de } \\
\text { seguidores }\end{array}$ \\
\hline 167.750 .765 & 5.424 .805 & 2.169 .560 & 3.255 .245 & 3.355 .015 \\
\hline
\end{tabular}

Tabla 1. Número de seguidores de los principales influencers de Clubhouse. Elaboración propia.

El número de usuarios totales que tiene el mayor influencer de la plataforma, Rohan Seth, es 5.424.805. Como se ha comentado, Rohan es cofundador de Clubhou- 
se. Llama la atención que tanto él, como Paul Davison (actualmente cuenta con 4.869.341 seguidores) sean los que más followers atesoran en su propia plataforma. Esta circunstancia no es común, ya que en las principales redes sociales del mundo son las celebrities de otros sectores las que ocupan los primeros puestos (We Are Social \& Hootsuite, 2021).

En cuanto al sector y cargo profesional que ostentan los 50 principales influencers se observa en la Figura 1 que la mayoría de ellos (34\%) son fundadores o directores generales de alguna empresa. El sector de las empresas a cargo de estos fundadores es muy variado: aceleradoras de pequeñas y medianas empresas, innovación y medioambiente, software e ingeniería, publicidad y marketing, fondos de capital riesgo, etc. En segundo lugar (26\%), se encuentran los artistas: músicos, actores y escritores. Entre ellos hay nombres conocidos mundialmente, como los actores Jared Leto (número 6 en seguidores con 4.263.562 followers) o Asthon Kutcher (número 42 en seguidores con 2.829.553 personas). Entre los músicos más seguidos se encuentra el guitarrista Bomani X (número 20 en seguidores con 3.405.303), quien fue la imagen de la aplicación durante los primeros meses de existencia (Rodriguez, 2021).

En tercer lugar están los influencers que son trabajadores o inversores de Clubhouse (14\%). Como se ha comentado anteriormente, los dos cofundadores también son los principales influencers de la red social. Además, entre los 50 primeros se encuentran algunos de los principales inversores que les respaldaron (France 24, 2021): Andrew Chen (número 12 en seguidores con 3.822.048 incondicionales) y Josh Constine (número 13 con 3.800 .790 admiradores).

Después se encuentran los inversores y economistas (12\%). Entre ellos hay varios gestores de fondos de capital riesgo, como Alexia Bonatsos (número 32 con 3.027.964 prosélitos) y Shondra Washington (número 33 con 3.002 .700 seguidores). Ambas mujeres invierten y manejan varias carteras de fondos de capital riesgo en la Bolsa estadounidense (Szkutak, 2021).

En quinto lugar se encuentran los influencers que se dedican a marketing y diseño (8\%). Entre ellos destacan Jasmine Martin (número 23 con 3.257.339 seguidores) y Swan Sit (número 29 con 3.058.157 discípulos). La segunda ha liderado el marketing digital de Nike, Revlon y Estée Lauder y en la actualidad, es asesora independiente del consejo de administración de varias empresas que cotizan en la Bolsa de Nueva York, además de ser socia operativa de AF Ventures y directora de marketing de Ani Energy, una nueva empresa de bebidas energéticas lanzada por las estrellas de TikTok, Josh Richards y Bryce Hall (SwanSit. com, 2021).

Por último, se encuentran los profesionales de otros sectores (6\%), como activistas o asesores de empresas. El primero de ellos es el activista Van Jones (número 9, con 3.921.116 seguidores), que trabajó como Consejero Especial para las áreas de empleo verde, empresa e innovación y calidad medioambiental durante la legislatura de Barack Obama. Hace unos meses, fue portada de varios periódicos de todo el mundo tras emocionarse con la victoria de Joe Biden ( $E l$ Confidencial, 2020). 


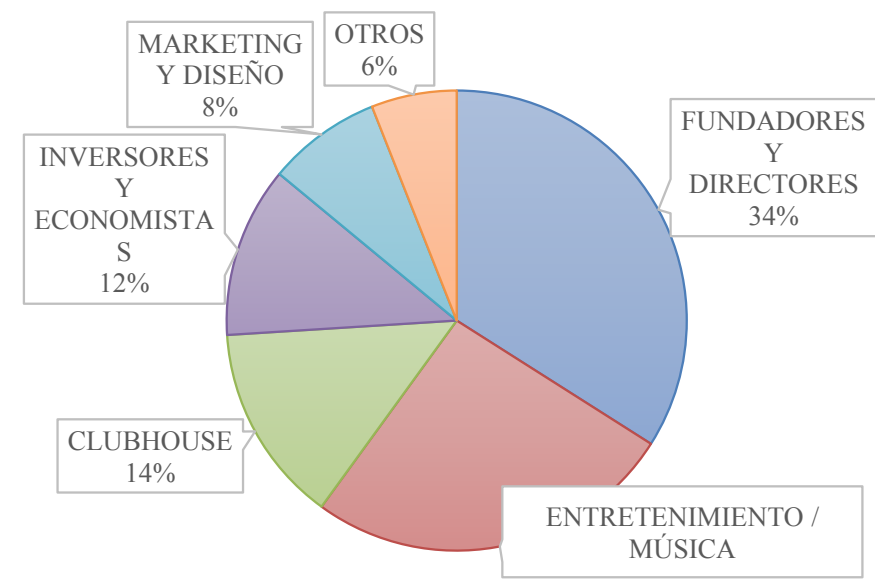

Figura 1. Cargo/sector profesional de los 50 principales influencers en Clubhouse. Elaboración propia.

En cuanto a la nacionalidad de los influencers, se observa que casi la totalidad de ellos son norteamericanos (estadounidenses y canadienses). Concretamente, 49 han crecido en el país norteamericano. La excepción es Erika Batista, que nació y se educó en la República Dominicana. Actualmente, es la directora jefe de la aceleradora de empresas de California, On Deck (Sifted, 2021).

Además de las categorías analizadas, se ha tratado de considerar la generación a la que pertenecen los influencers de éxito en Clubhouse. Como se puede observar en la Figura 2, el 50\% son millennials (nacidos entre los años 1981 y 1993); es decir, tienen entre 28 y 40 años en estos momentos. En segundo lugar, con un $32 \%$, se encuentran los nacidos entre 1969 y 1980 o Generación X. Posteriormente, los Baby Boomers, nacidos entre 1949 y 1968 , que suman un $10 \%$. Por último, hay un $8 \%$ de influencers más jóvenes que los anteriores, son los denominados Generación $\mathrm{Z}$ o centennials (nacidos entre 1994 y 2010) que tienen menos de 28 años en la actualidad.

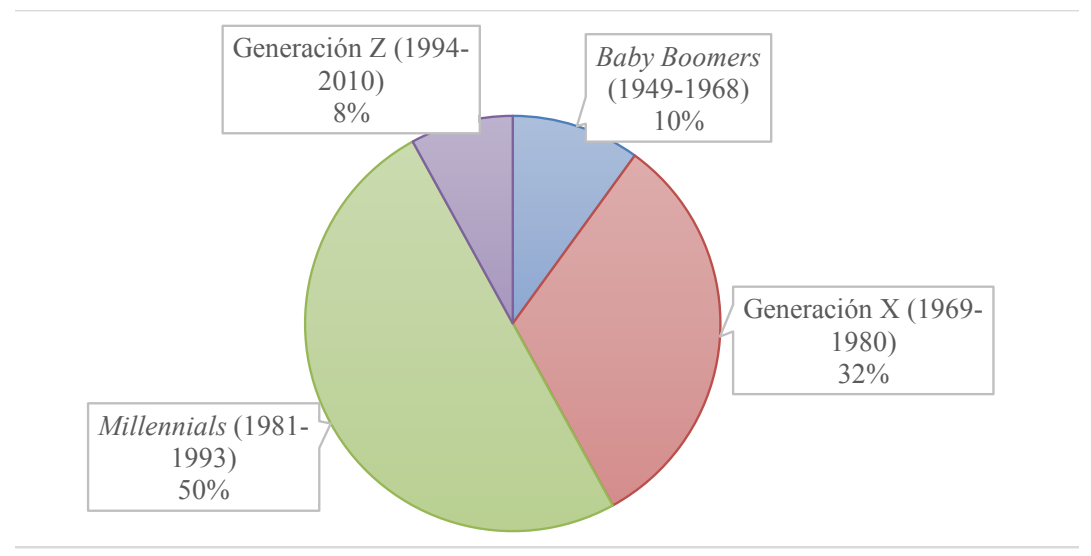

Figura 2. Generación a la que pertenecen los 50 principales influencers en Clubhouse. Elaboración propia. 
En cuanto a la representación de hombres y mujeres, entre los principales influencers de Clubhouse, se observa en la Figura 3 que hay una mayor representación de los primeros $(62 \%)$ frente a las segundas $(38 \%)$ :

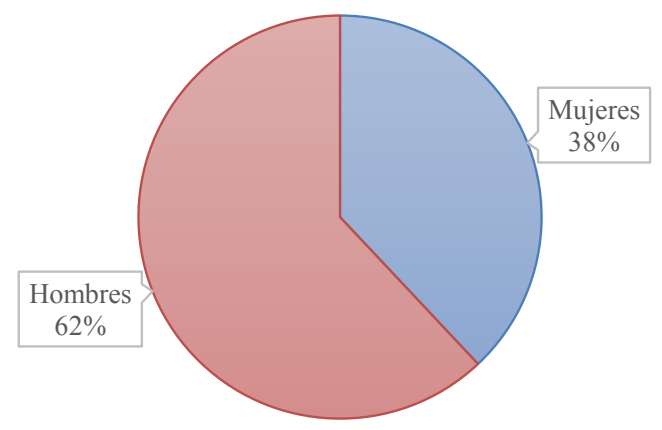

Figura 3. Hombres y mujeres entre los 50 principales influencers en Clubhouse.

Elaboración propia.

Si se comparan los seguidores acumulados por sexo, se observa que los varones acumulan un total de 105.287.987 de followers, frente a los 62.462 .778 de acólitos que tienen las mujeres (ver Tabla 2).

Tabla 2. Total de seguidores por sexo de los 50 principales influencers en Clubhouse. Elaboración propia.

\begin{tabular}{|c|c|}
\hline Total seguidores influencers hombres & Total seguidores influencers mujeres \\
\hline 105.287 .987 & 62.462 .778 \\
\hline
\end{tabular}

Es un dato llamativo que habrá que estudiar en los próximos meses, cuando la red social esté más asentada y existan más métricas de análisis.

\section{Conclusiones, discusión y prospectivas}

Esta investigación proponía ahondar en las claves de Clubhouse, sus usos y sus posibilidades a través de una metodología correlativa en dos fases. La investigación bibliográfica ha validado la originalidad de este estudio. Son numerosas y sugestivas las investigaciones científicas sobre redes sociales, el compromiso que generan y la relación que establecen con distintas audiencias. Sin embargo, debido a la inexistencia de alguna API (Application Programming Interface) para medir los parámetros de funcionamiento de la red social, todavía no existe tradición académica sobre Clubhouse, aunque los medios de comunicación ya le han dedicado muchos artículos de análisis.

La segunda parte de la metodología, el análisis cuantitativo de los 50 usuarios con más seguidores en Clubhouse, a 31 de marzo de 2021, ha arrojado varias ideas 
interesantes: el usuario con más seguidores en Rohan Seth, uno de los dos creadores, con más de 5 millones de followers; el 34\% de los 50 primeros influencers de Clubhouse son fundadores o directores generales de alguna empresa; los 49 primeros de la lista son norteamericanos (estadounidenses o canadienses, como Elon Musk) y la excepción es una mujer dominicana, que trabaja en California.

Es interesante destacar que al haber un alto porcentaje de altos cargos se abre la oportunidad de crear una experiencia social que se sienta más humana. Al romperse esta barrera y acceder los propios directores y fundadores, existe una nueva cercanía entre los usuarios habituales de la red social y los creadores de contenido de la plataforma.

Estas ideas plantean algunos retos para la plataforma y varias prospectivas de investigación para los académicos. En primer lugar, se valora que Clubhouse haya propuesto una alternativa a las redes sociales que se centran en la imagen, con los peligros que ello implica, y en algoritmos demasiado centrados en los likes. Esta red social ni siquiera cuenta con una posibilidad similar a un 'me gusta' y sencillamente, pregunta a los usuarios sus intereses al registrarse, subrayando que esos intereses pueden modificarse cuantas veces se desee. Esta circunstancia invita a pensar y comparar las formas de medición del compromiso en redes sociales, y cómo Clubhouse y TikTok están ofreciendo formas muy contrarias a Facebook, Twitter o Instagram.

Por otro lado, Clubhouse se basa en el audio, en forma de charlas variadas y numerosas, a todas horas del día y desde cualquier parte del mundo. Cualquier usuario puede unirse a la sala y hablar con el presentador y los demás usuarios, tras pedir la palabra levantando la mano. Lo que ocurre en esa sala no queda grabado, a no ser que Clubhouse así lo decida. En cualquier caso, no lo hace público y prohíbe a los usuarios grabar los encuentros. Se defiende un audio efímero e íntimo, que deja una huella emocional y directa en los participantes. Estos detalles podrían ser objeto de estudio de futuras investigaciones, valorando el efecto de determinadas charlas en grupos concretos de usuarios que podrían ser después entrevistados: ¿por qué eligen Clubhouse?, ¿por qué eligen ese tema?, ¿por qué siguen a ese influencer?, ¿ese tema o ese influencer deberían tener más cobertura y visibilidad en los medios?, ¿qué otras actividades se dejan de realizar mientras se dedica ese tiempo a Clubhouse?

Asimismo, hemos conocido el valor actual de la compañía y el crecimiento exponencial de su número de usuarios. A partir de ahí, nos podemos preguntar cómo lo valora la competencia. Los usuarios de Twitter ya pueden entrar a sus propias salas gracias a Twitter Spaces, aunque se desconoce qué porcentaje de los más de 190 millones de usuarios de la red de microblogging accede asiduamente a esta nueva opción de audio. ¿Qué harán las otras redes sociales?, ¿tienen espacio para el audio?, ¿Facebook implementará pronto algo parecido, tal como aventuran los medios? o por el contrario, ¿puede Clubhouse decaer ante el poder de WhatsApp, la primera red social del mundo, si ésta inventa una herramienta semejante?

Cada pregunta puede dar lugar a nuevas investigaciones y sólo está claro, por ahora, que hay que estar atentos a la dinámica que se genera en las salas de audio, ya que los usuarios de Twitter se confrontan de manera directa con los tuits y las salas de Clubhouse podrían convertirse en espacios ampliados para esos enfrentamientos. Siendo así, ¿las salas de audio podrían convertirse en nuevos espacios para la argumentación y el debate, más allá de lo que permite una publicación puntual en una red social? o ¿Clubhouse ha de ampliar rápidamente sus normas comunitarias de cara a moderar lo que ocurra en cada sala? 
Cuando esté completamente disponible para el sistema operativo Android en todos los países y funcione sin invitaciones de usuarios, Clubhouse tendrá que validar su espacio propio, más allá de la COVID-19 y sus limitaciones técnicas, remarcando lo que ahora parecen sus fortalezas: no importan la imagen ni los likes, ofrece salas de debate íntimas, con discusiones no medidas sobre temas de actualidad con líderes de opinión e influencers, y promueve la voz como herramienta básica, accesible y universal contra la brecha digital.

\section{Referencias Bibliográficas}

Abelairas-Etxebarria, P. y Mentxaka Arana, J. (2020). ¿Qué opinan los universitarios sobre el uso académico de las redes sociales? Revista Interuniversitaria de Investigación En Tecnología Educativa, (8), 1-12. https://doi.org/10.6018/riite.362121

Aladro-Vico, E., Jivkova-Semova, D. \& Bailey, O. (2018). Artivism: A new educative language for transformative social action. Comunicar, 57, 9-18. https://doi.org/10.3916/ C57-2018-01

Al-Regaiey, K. A.; Alshamry, W. S; Alqarni, R. A.; Albarrak, M. K.; Alghoraiby, R. M.; Alkadi, D. Y.; Alhakeem, L. R.; Bashir, S. \& Iqbal, M. (2021). Influence of social media on parents' attitudes towards vaccine administrativo. Human Vaccines \& Immunotherapeutics, online, 1-18. https://doi.org/10.1080/21645515.2021.1872340

Asociación para la Investigación de Medios de Comunicación (AIMC, 2021). 23 ${ }^{\circ}$ Informe Navegantes en Red. Recuperado de: http://download.aimc.es/aimc/cc8ke5T/ macro2020/\#page $=1$

Bai, J; Li, H. \& Chen, J. (2020). Multiple Star Node Discovery Algorithm in Social Network Based on Six Degrees of Separation and Greedy Strategy. En: V. Jain, S. Patnaik, F. Popențiu Vlădicescu \& I. K. Sethi (Eds.), Recent trends in intelligent computing, communication and devices. Advances in intelligent systems and computing, pp. 623-632. Nueva York. Springer. Recuperado de: https://doi.org/10.1007/978-981-13-9406-5_74

Barbeito Veloso, M. L. y Perona Páez, J. J. (2020). Emisoras infantiles en internet. Análisis de una oferta alternativa para el niño-oyente. Icono14, 16(2), 100-120. https://doi. org/10.7195/RI14.V16I2.1180

Barenys, M. y Expósito, D. (2021, 6 de marzo). ClubHouse, la app dispuesta a redimensionar las redes sociales: "Es como el Twitter de la voz". Nius Diario. Recuperado de: https:// tinyurl.com/yfsvtmm9

BBC News Mundo (2021, 22 de febrero). Clubhouse: ¿qué es esta aplicación y cómo se obtiene una invitación? Recuperado de: https://tinyurl.com/pn7nvx29

Bentley, K.; Chu, Ch.; Nistor, C.; Pehlivan, E. \& Yalcin, T. (2021). Social media engagement for global influencers. Journal of Global Marketing, online, 1-15. https://doi.org/10.108 0/08911762.2021.1895403

Bernárdez-Rodal, A.; Padilla-Castillo, G. y Sosa Sánchez, R. P. (2019). From Action Art to Artivism on Twitter and Instagram: relocation and instantaneity for a new geography of protest. Catalan Journal of Communication \& Cultural Studies, 11.1, 23-37. Doi: 10.1386/cjes.11.1.23_1

Caldevilla, D.; Barrientos, A. y Padilla, G. (2021). Twitter as a Tool for Citizen Education and Sustainable Cities after COVID-19. Sustainability, 13, 3514. https://doi.org/10.3390/ su13063514 
Calvo-Gutiérrez, E. y Padilla-Castillo, G. (2011). Radio 3.0. Éxito durmiente. En M. A. Ortiz Sobrino y N. López Vidales (Coords.), Radio 3.0. Una nueva radio para una nueva era. Madrid, Fragua, pp. 281-300.

Chiza-Lozano, D.; Vásquez-Mendoza, D. \& Ramírez Vega, C. (2021). Adicción a redes sociales y ciberbullying en los adolescentes. Revista Muro de La Investigación, 6(1), 3444. https://doi.org/10.17162/rmi.v6i1.1437

El-Khoury, J.; Haidar, R.; Kanj, R. R.; Bou Ali, L. \& Majari, G. (2021) Characteristics of social media 'detoxification' in university students. Libyan Journal of Medicine, 16(1). https://doi.org/10.1080/19932820.2020.1846861

France 24 (2021, 26 de enero). Red social de audio Clubhouse prepara lanzamiento masivo. Recuperado de: https://www.france24.com/es/minuto-a-minuto/20210125-red-social-deaudio-clubhouse-prepara-lanzamiento-masivo

Grier, F. (2019). Musicality in the consulting room. The International Journal of Psychoanalysis, 100(5), 827-851. https://doi.org/10.1080/00207578.2019.1664905

Hogan, B. (2021, 19 de febrero). Clubhouse Statistics. SoftwarePundit. Recuperado de: https://tinyurl.com/abp73swx

IAB Spain (2020a). Estudio de Redes Sociales 2020. Recuperado de: https://iabspain.es/ estudio/estudio-redes-sociales-2020

IAB Spain (2020b). Top Tendencias Digitales 2020. Recuperado de: https://iabspain.es/ estudio/top-tendencias-digitales-2021/

Lewin, A. (2021, 17 marzo). What is On Deck? And is it really as magic as people say it is? Sifted. https://sifted.eu/articles/on-deck-fellowship-europe/

López Fuentes, D. D. (2020). Impacto de las redes sociales en el entorno educativo. Ciencia, Cultura y Sociedad, 6(1), 73-77. https://doi.org/10.5377/ccs.v6i1.10538

Lorenzo, A. (2021, 7 de febrero). 15 cosas que debes saber sobre 'Clubhouse', la red social que más dará que hablar. El Economista. Recuperado de: https://www.eleconomista.es/ tecnologia/noticias/11036166/02/21/15-cosas-que-debes-saber-sobre-Clubhouse-la-redsocial-que-mas-dara-que-hablar.html

Marinas, L. (2019). Instagram: Donde Millennials, Generación Z, Mcluhan y Bolter se cruzan. CIC Cuadernos De Información y Comunicación, 24, 187-201. https://doi.org/10.5209/ ciyc. 64641

Mason, A. N.; Narcum, J. \& Mason, K. (2021). Social media marketing gains importance after Covid-19. Cogent Business \& Management, 8(1), online. https://doi.org/10.1080/2 3311975.2020 .1870797

Matassi, M. \& Boczkowski, P. J. (2020). Redes sociales en Iberoamérica. El Profesional de La Información, 29(1), 1-21. https://doi.org/10.3145/epi.2020.ene.04

Mills, M.; Stoneham, G. \& Georgiadou, I. (2017). Expanding the evidence: Developments and innovations in clinical practice, training and competency within voice and communication therapy for trans and gender diverse people. International Journal of Transgenderism, 18(3), 328-342. https://doi.org/10.1080/15532739.2017.1329049

Moreno, M. (2020, 7 de noviembre). Un colaborador de la CNN se derrumba en directo tras la victoria de Joe Biden en Estados Unidos. El Confidencial. https://www.elconfidencial. com/television/programas-tv/2020-11-07/cnn-van-jones-victora-joe-biden-eleccioneseeuu_2823951/

Ortega, E. y Rodríguez, J. (2021). Estrategia de comunicación de los cuerpos de seguridad a través de píldoras audiovisuales en TikTok. Policía Nacional y Guardia Civil en España. Revista Internacional de Investigación en Comunicación aDResearch ESIC, (25), 160 185. https://doi.org/10.7263/adresic-025-09 
Padilla-Castillo, G. (2016). Redes sociales institucionales de éxito: los casos de @policia y@guardiacivil en España. Revista Opción, 32(12), 85-109. Recuperado de: http:// produccioncientificaluz.org/index.php/opcion/article/view/22038/21761

Padilla-Castillo, G. (2021). TikTok como vía de promulgación de la fe católica. En Comunicación a la vanguardia. En Tendencias, métodos y perspectivas. ISBN: 978-847074-899-8. Madrid, España. Editorial Dykinson.

Paredes, L. M. (2021, 11 de febrero). Qué es Clubhouse y por qué se ha convertido en la app del momento. Digitaltrends. Recuperado de: https://es.digitaltrends.com/guias/quees-clubhouse/

Parra, S. (2011, 3 de mayo). El podcast de 1889, el primer podcast de la historia. Xataka Ciencia. Recuperado de: https://www.xatakaciencia.com/tecnologia/el-podcast-de-1889el-primer-podcast-de-la-historia

Pedrero-Esteban, L. M. y Pérez-Alaejos, M. de la P. M. (2017). La transición de la radio al entorno digital: experiencias y retos transmedia de la industria radiofónica española. Novos Olhares, 6(2), 41-51. DOI: 10.11606/issn.2238-7714.no.2017.134668

Pedrero-Esteban, L., y García Lastra, J. (Coord.) (2019). La transición digital de la radio. Diez claves para su comprensión profesional y académica. Valencia, España. Tirant Humanidades.

Piñeiro-Otero, T. (2021a). Entre la sensación y la contención. La multi-dimensionalidad de la escucha en Inside. L'Atalante. Revista de estudios cinematográficos, 31, 103-116. Recuperado de: http://www.revistaatalante.com/index.php?journal=atalante\&page=articl e\&op=view\&path $\% 5 \mathrm{~B} \% 5 \mathrm{D}=878 \&$ path $\% 5 \mathrm{~B} \% 5 \mathrm{D}=642$

Piñeiro-Otero, Teresa (2021b). Los nuevos modelos de publicidad en revistas nativas digitales deportivas: el caso de Marca Plus. Revista Inclusiones, 8, 231-254. Recuperado de: http://revistainclusiones.com/carga/wp-content/uploads/2021/03/12-Pineiro-EspanaCongreso-VOL-8-NUM-AbrilJunoo2021INCL.pdf

Ponce, I. (2012, 17 de abril). Redes Sociales. Historia de las redes sociales. Monográfico: Redes Sociales. Madrid, España. Ministerio de Educación, Cultura y Deporte. Recuperado de: http:// recursostic.educacion.es/observatorio/web/en/internet/web-20/1043-redes-sociales?start=2

Quinn, P.; McGilloway, S. \& Burke, J. (2021). COVID-19 and the class of 2020: a national study of the mental health and wellbeing of Leaving Certificate students in Ireland. Irish Educational Studies, online, 1-10. https://www.tandfonline.com/doi/abs/10.1080/033233 15.2021.1916564

Rimoldi, E. (2020). Futuröck: primera radio profesional por internet en Argentina. Question/ Cuestión, 2(66), online. https://doi.org/10.24215/16696581e430

Rodriguez, S. (2021, 10 de enero). How Black users are saving Clubhouse from becoming a drab hangout for tech bros. CNBC. Recuperado de: https://www.cnbc.com/2021/01/10/ black-users-turned-social-app-clubhouse-from-drab-to-fun.html

Stevenson, S. (2021). Learning from COVID-19: Revisiting Organizational Communications from the Bottom-up. Public Library Quarterly, online, 1-16. https://www.tandfonline. com/doi/abs/10.1080/01616846.2021.1906616

Szkutak, R. (2021, 17 marzo). This Group Of 150-Plus Women Investors Is Looking To Level Access To Capital For Emerging VCs. Forbes. Recuperado de: https://www.forbes. com/sites/rebeccaszkutak/2021/03/17/how-transact-global-helps-emerging-women-vcsland-lp-commitments/

SwanSi.com (2021). What does Swan do?. Recuperado de: https://www.swansit.com/

Toffler, A. (1979). La Tercera Ola. Nueva York, Estados Unidos. Bantam Books. 
Verwey, S. (2015). Self-expression and collaborative 'pro-sumption' in the digital brandscape. Communicatio, 41(3), 320-339, https://doi.org/10.1080/02500167.2015.1093327

Videla Rodríguez, J. J. y Piñeiro-Otero, T. (2017). La radio online y offline desde la perspectiva de sus oyentes-usuarios. Hacia un consumo híbrido. Estudios sobre el Mensaje Periodístico, 23(2), 1437-1455. https://doi.org/10.5209/ESMP.58054

We Are Social \& Hootsuite (2020). Digital 2020. Recuperado de: https://wearesocial.com/ blog/2020/04/digital-around-the-world-in-april-2020 